\title{
Planification du retrait des fonds du pilier 3a
}

Nombre de personnes actives utilisent les avantages du pilier 3a en déduisant chaque année les primes sur leur déclaration de revenu. Une économie d'impôts peut non seulement être réalisée pendant la durée de l'assurance mais également lors de son versement. A la retraite les fonds du pilier 3a sont transférés de la fortune liée à l'avoir libre ce qui a pour conséquence une imposition unique du capital. Celle-ci est progressive comme la plupart des taux d'imposition sur le revenu. Cela veut dire que plus la fortune est élevée plus le taux d'imposition sera élevé. Ainsi toutes les prestations de la retraite, à savoir les versements du pilier $3 a$ et les prestations du capital de la prévoyance professionnelle, sont additionnées durant l'année en cours et imposées en commun.

De ce fait, une bonne planification débute de nombreuses années avant la retraite. Au plus tard cinq ans avant la retraite officielle les versements devraient être planifiés, afin de choisir différentes années pour le versement. L'exemple ci-dessous illustre les économies fiscales que vous pouvez réaliser:

\begin{tabular}{|c|c|c|c|c|c|c|c|c|c|c|c|}
\hline Canton* & $1 \times 400000$ & $2 \times 200000$ & Economie & Canton* & $1 \times 400000$ & $2 \times 200000$ & Economie & Canton* & $1 \times 400000$ & $2 \times 200000$ & Economie \\
\hline AG & 39234 & 31618 & $19,41 \%$ & GR & 19473 & 17146 & $11,95 \%$ & SZ & 23693 & 13910 & $41,29 \%$ \\
\hline $\mathrm{Al}$ & 68553 & 66226 & $3,39 \%$ & JU & 32896 & 28874 & $12,23 \%$ & TG & 30713 & 28386 & $7,58 \%$ \\
\hline$A R$ & 31473 & 29146 & $7,39 \%$ & LU & 34455 & 28234 & $18,06 \%$ & $\mathrm{TI}$ & 23833 & 21506 & $9,76 \%$ \\
\hline BE & 35814 & 28722 & $19,80 \%$ & NE & 37508 & 35182 & $6,20 \%$ & UR & 24230 & 19036 & $21,44 \%$ \\
\hline$B L$ & 22632 & 19106 & $15,58 \%$ & NW & 32923 & 27686 & $15,91 \%$ & VD & 43812 & 34838 & $20,48 \%$ \\
\hline BS & 36823 & 31246 & $15,15 \%$ & OW & 29817 & 27490 & $7,80 \%$ & VS & 32540 & 23144 & $28,88 \%$ \\
\hline FR & 46657 & 37962 & $18,64 \%$ & SG & 31745 & 25114 & $20,89 \%$ & ZG & 25374 & 18658 & $26,47 \%$ \\
\hline GE & 27695 & 22152 & $20,01 \%$ & SH & $25^{\prime} 735$ & 20274 & $21,22 \%$ & $\mathrm{ZH}$ & 30538 & 24066 & $21,19 \%$ \\
\hline GL & 33073 & 29146 & $11,87 \%$ & so & $32^{\prime} 139$ & 26278 & $18,24 \%$ & & & & \\
\hline
\end{tabular}

* Les capitales cantonales ont été retenues pour l'impôt communal.

Solutions pilier 3a pour médecins

Nous collaborons avec la majorité des compagnies d'assurance et diverses banques afin d'être en mesure de vous soumettre les meilleures solutions. De plus, nous disposons de produits spéciaux qui vous permettent d'obtenir des avantages supplémentaires exclusivement par FMH Insurance Services. Nos conseillers sont volontiers à votre disposition pour de plus amples informations ou pour vous soumettre une offre personnalisée.

\section{Talon-réponse}

Prénom/Nom

Adresse

NPA/Lieu

Date de naissance

Téléphone privé/ cabinet

Atteignable le plus facilement (heure)

Adresse e-mail

O Je désire optimiser fiscalement le retrait des montants de ma prévoyance. Prière de me téléphoner.

- Je désire apprendre à connaître les solutions du 3 e pilier pour médecins. Prière de me téléphoner.

O Je désire connaître toutes les autres prestations de services de FMH Insurance Services.

Prière de me téléphoner.

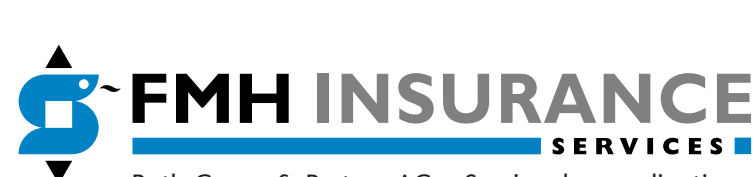

Roth Gygax \& Partner AG $\square$ Service de coordination Moosstrasse 2 - 3073 Gümligen

Téléphone 0319595000 — Fax 0319595010 mail@fmhinsurance.ch @ www.fmhinsurance.ch 